\title{
Selective Neurodegeneration in Huntington's Disease
}

In this issue of the Annals of Neuralogy, Richfield and colleagues [1] publish the latest in a series of reports describing the preferential loss of a specific population of striatal neurons in Huntington's disease (HD). While careful neuropathological analyses have demonstrated neuron loss in many brain regions, the striatum is disproportionately affected in HD and work from several groups has now resulted in a fairly detailed understanding of the pattern of striatal neurodegeneration in HD. This achievement was made possible by the recognition and description of diverse subpopulations of striatal neurons.

Striatal neuron subpopulations are divided into two broad categories: interneurons whose axonal arborizations stay within the striatum, and projection neurons with axons terminating in the pallidum or substantia nigra. Striatal projection neurons comprise the vast majority of striatal neurons, perhaps $90 \%$ in all, while there are several different subpopulations of striatal interneurons, each comprising a small fraction of striatal neurons. Three subpopulations of striatal interneurons are particularly well characterized: one subpopulation expressing somatostatin (SS), neuropeptide $\mathrm{Y}$ (NPY), and nitric oxide synthase (NOS); a second subpopulation expressing acetylcholine; and a third $\gamma$ aminobutyric acid (GABA)ergic subpopulation expressing the calcium-binding protein parvalbumin. Studies of striatal interneurons provided the first evidence that not all striatal neurons are equally affected in HD. In 1985, work by two groups [2, 3] demonstrated that striatal SS/NPY/NOS neurons are at least relatively spared in HD. It was subsequently appreciated that while markers of striatal cholinergic function decline in HD, there is preservation of striatal cholinergic perikarya, indicating functional impairment but not degeneration of striatal cholinergic interneurons [4]. Harrington and Kowall [5] suggested that striatal $\mathrm{GABA}$ /parvalbumin-containing interneurons are preserved early in the course of HD but degenerate with progression of HD.

Striatal projection neurons are subdivided into subpopulations on the basis of their target regions and which neuropeptides they contain. Striatal projection neurons terminate in either the external segment of the globus pallidus $(\mathrm{GPe})$, the internal segment of the globus pallidus (GPi), the substantia nigra pars reticulata $(\mathrm{SNr})$, or the substantia nigra pars compacta $(\mathrm{SNc})$ with few collaterals to other target regions. All striatal projection neurons are GABAergic but striato-GPe neurons express enkephalins while striato-GPi, striato$\mathrm{SNr}$, and striato-SNc neurons express tachykinins such as substance $\mathrm{P}$. Initial findings by Reiner and coworkers [6], now confirmed by Richfield and colleagues [1] and other groups, indicated that striato-GPe and striato-SNr neurons degenerate preferentially early in the course of $\mathrm{HD}$, with striato-GPi neurons relatively spared early in the course of HD. Remarkably, loss of striato-GPe neurons has been observed in presymptomatic $\mathrm{HD}[7]$. The early and relatively selective loss of striato-GPe and striato-SNr neurons is a plausible explanation for the chorea and oculomotor abnormalities that are prominent clinical features of early HD. Hedreen and Folstein [8] suggested recently that striato-SNc neurons are affected even earlier in the course of HD than are striato-GPe neurons. While awaiting confirmation, this observation would complete an inferred model of disease progression in which striatal projection neurons degenerate in a specific temporal sequence: striato-SNc followed by striato-GPe and striato-SNr, then followed by striato-GPi. Striatal GABA/ parvalbumin interneurons also degenerate with relative sparing, through functional impairment, of striatal cholinergic interneurons and preservation of striatal SS/ NPY/NOS interneurons. Striatal afferent terminals, such as those of the nigrostriatal projection, appear to be relatively spared in $\mathrm{HD}$.

Knowledge of affected and spared striatal neuron subpopulations proved invaluable in assessing putative models of HD. The reproduction of many features of HD striatal pathology by intrastriatal injections of $\mathrm{N}$-methyl-D-aspartate (NMDA) receptor agonists is strong evidence for an NMDA receptor/excitotoxic mechanism of neuronal degeneration in HD. The NMDA hypothesis is a rational basis for clinical experiments and is the point of departure for a large clinical trial being prepared by the Huntington's Study Group.

The differences between brain regions and between striatal neuron subpopulations in their susceptibility to the pathogenetic process caused by abnormal HD alleles is in marked contrast to the wide expression of the HD gene product [9]. Both in situ hybridization studies of messenger RNA expression and immunohistochemical studies of the protein, huntingtin, encoded by the HD gene have shown uniform expression of this gene in all neurons examined and in several peripheral tissues. The abnormal gene product, characterized by expanded polyglutamine repeats near the amino terminus, is also expressed. The unique pattern of pathology 
found in HD cannot be accounted for by the pattern of expression of huntingtin, and additional factors specific to the affected neurons must account for the differential degeneration or survival of striatal and other neuron subpopulations. Intensive investigation of huntingtin is now under way and it is hoped that further characterization of this protein will lead to clues regarding the mechanism(s) of neurodegeneration. Some evidence, however, suggests that the abnormal gene product acts by a so-called gain of function effect in which the abnormal allele has gained a new and pathological effect, possibly unrelated to the function of the normal protein [9]. If so, discovery of the normal function of huntingtin may not cast light on the mechanism(s) of neuronal death. In any case, huntingtin has little homology to known proteins and characterization of huntingtin function(s) may be an arduous process.

Further investigation of the biology of striatal neurons should be pursued as a parallel approach to uncovering clues regarding neurodegeneration in HD. The clear differences in susceptibility among striatal neuron subpopulations suggest that broad-based investigation of the properties of striatal neuron subpopulations might reveal crucial differences between affected and spared striatal neuron subpopulations. To develop scientific strategies for arresting progression of $\mathrm{HD}$, we need to understand the interplay between the abnormal gene product and the specific factors governing neuronal susceptibility. We may ultimately achieve a comprehensive understanding of neuronal degenera- tion in HD that will assist in understanding the mechanisms of neurodegeneration in other disorders.

Roger L. Albin, MD

Department of Neurology

University of Michigan

Ann Arbor, MI

\section{References}

1. Richfield EK, Maguire-Zeiss KA, Vonkeman HE, Voorn P. Preferential loss of preproenkephalin versus preprotachykinin neurons from the striatum of Huntington's disease patients. Ann Neurol 1995;38:852-861

2. Ferrante RJ, et al. Selective sparing of a class of striatal neurons in Huntington's disease. Science 1985;230:561-564

3. Dawburn D, Dequidt ME, Emson PC. Survival of basal ganglia neuropeptide $Y$-somatostatin neurones in Huntington's disease. Brain Res 1985;340:251-260

4. Hirsch EC, Graybiel AM, Hersh LB, et al. Striosomes and extrastriosomal matrix contain different amounts of immunoreactive choline acetyltransferase in the human striatum. Neurosci Lett 1989;96:145-150

5. Harrington KM, Kowall NW. Parvalbumin immunoreactive neurons resist degeneration in Huntington's disease striatum. J Neuropathol Exp Neurol 1991;50:309

6. Reiner A, et al. Differential loss of striatal projection neurons in Huntington disease. Proc Natl Acad Sci USA 1988;85:57335737

7. Albin RL, et al. Preferential loss of striato-external pallidal projection neurons in presymptomatic Huntington's disease. Ann Neurol 1992;31:425-430

8. Hedreen JC, Folstein SE. Early loss of striosome neurons in Huntington's disease. J Neuropathol Exp Neurol 1995;54:105-120

9. Albin RL, Tagle DA. Genetics and molecular biology of Huntington's disease. Trends Neurosci 1995;18:11-14 